\title{
OBSTETRIC CHOLESTASIS
}

Pradhan $\mathbf{P}^{1}$

\section{ABSTRACT}

Obstetric Cholestasis is the commonest liver disease that causes pruritis and is unique to pregnancy. Pruritis can be so intense as to lead insomnia.The Significance of this disease has been highlighted more recently due to the associated perinatal mortality and maternal morbidity. Aetiology and pathophysiology still uncertain. There, appears to be genetic predisposition in certain individuals resulting in an increased susceptibility to the high oestrogen levels found in pregnancy specially in $3^{\text {rd }}$ trimester and resolving promptly after delivery when oestrogen level falls rapidly. Pruritis is central in origin and thus fails to respond to commonly used antihistamines and lotio calamine locally. Recently ursodeoxycholic acid an exogenous bile acid is increasingly being used and showed improved both pruritis and liver function and favourable changes were observed in the foetus as well. Delivery planned at 37-38 weeks of gestation reduced perinatal mortality. Because of increased rate of adverse intrapartum events, close monitoring is appropriate. Active management of the third stage is necessary because of the increased risk of post partum haemorrhage.

Key Words: Pruritis, pregnancy, planned delivery, perinatal mortality and maternal morbidity.

\section{INTRODUCTION}

Obstetric Cholestasis also called intrahepatic cholestasis of pregnancy (ICP) is the commonest liver disease that is unique to pregnancy. It is second only to viral hepatitis as the leading cause of Jaundice in pregnancy. It was first reported by Ahfiled in 1883 and not until 1950 that several clinical case reports begin to emerge. ${ }^{1}$ Its significance has been highlighted more recently due to the associated perinatal Mortality and maternal morbidity with this condition. Aetiology and pathophysiology of this condition remains uncertain and there is lack of consensus regarding appropriate methods of fetal surveillance.

1. Nepal Medical College Teaching Hospital, Jorpati, Kathmandu, Nepal.

Address for correspondence : Prof. Dr. Pramila Pradhan

Dept. of Obstetrics / Gynaecology

Nepal Medcial College Teaching Hospital

Jorpati, Kathmandu, Nepal

Tel: 253573 


\section{DEMOGRAPHY}

The prevalence of obstetric cholestasis varies throughout the world. The highest rate have been reported in south America particularly in Chile at $12-22 \%^{2}$ Scandinavia has reported rates of $2 \%^{3}$ and European reported in the region of $0.2 \%{ }^{4}$. The prevalence in India and Nepal is not known. Racial variation has been reported, incidence being higher in native South American Indians. ${ }^{2,5}$ Seasonal variation is also seen with higher rates in the winter months. However comparison between series has been limited by variation in diagnostic criteria and socio-economic factors. Incidence appear to be increasing although this may be secondary to increased awareness and therefore increased case ascertainment.

\section{AETIOLOGY AND PATHOGENESIS}

The exact aetiology is unknown. Genetic studies have revealed a positive family history in $33-50 \%$ of patients and there have been suggestions of an Autosomal dominant inheritance pattern ${ }^{6,7}$. The condition is known to recur in $40-45 \%$ of pregnancy. ${ }^{8}$ Thepathogenesis of obstetric Cholestasis is multifactorial. Oestrogen appear to be the main factor involved. There appear to be genetic predisposition in certain individuals resulting in an increased susceptibility to the high estrogen levels found in pregnancy .Level of oestrogen are highest in third trimester and this is the most common time of presentation. This leads to an impaired ability of hepatocytes to conjugate and excrete hormone metabolites as well as bile acids both of which share a similarity of steroid structure.

The intensity of cholestasis has been shown to parallel the blood oestrogen levels peaking in late pregnancy and resolving promptly after delivery when oestrogen level falls rapidly. Cholestasis is in fact is a known complication of oestrogen therapy in humans and has been produced in rats treated with high doses of this hormone. ${ }^{9}$ Furthermore obstetric cholestasis resembles a similar condition that some women develop while taking the combined oral contraceptive pills. Obstetric cholestasis is more common in twin pregnancies it is probably an increased sensitivity to oestrogen rather than the actual levels of oestrogen that is important. ${ }^{10}$ The mechanisn of oestrogen is unclear but it appear to involve disruption of the membrane transport mechanisn across the biliary canalicular membrane, resulting in a functional obstruction with resultant into the plasma of biliary constituents, such as bilirubin and alkaline phosphatase. This leads to an alteration of the cholesterol. Phospholipid ratio. ${ }^{11,12}$ This condition is characterised by elevated concentration of total bile acids (TBA) in the serum to level>11.0 umol/L and by a higher proportion of toxic hydrophilic bile acid than the non toxic hydrophilic ones. Altered bile acid ratio of cholic acid (CA): Cheriodeoxy Cholic acid (CDA) of $>1.5$, elevated proportion of CA Constituting $>42 \%$ of the total bile acids and a decrease proportion of gycine conjugated bile acids compared with the taurine conjugated ones $(<1.0)$ are other findings. ${ }^{13}$ Cholic acid is the primary pathogenic agent which is also known to cross the placental barrier with the attendant problems of increased morbidity and mortality. ${ }^{14}$

\section{CLINICAL FEATURES}

The main symptom is severe pruritis. This usually develops in the third trimester of pregnancy generally after 30 weeks of gestations and is relieved promptly within few days to 4 weeks after delivery. ${ }^{8}$ Classically it develops on the soles of feet, palms of hands and spread to trunk and limbs. It may be so intense as to lead to insomnia. There is no associated skin rash but excoriation may be 
present .Jaundice is less common and develops two to three weeks after the onset of priritis. If present it reflects more advanced disease. There may also be other symptoms such as dark urine, pale stool, anorexia and steatorrhoea with fat malabsorption as a result. Bile salts and vit.K deficiency may result. There is usually a strong family history of this disorder. A similar history of pruritis in previous pregnancies in a multiparous women is usually forthcoming.

The exact cause of the pruritis is unknown .Deposition of bile acids and histamine release is one theory although an increase in central nervous system opiates receptors has also been proposed. ${ }^{15}$ Other cause of Jaundice and cholestasis need to be considered in the differential diagnosis and excluded by appropriate investigations. These include acute vital hepatisis, drug induced jaundice, sepsis, biliary stone disease and other rarer causes. Assessment of liver functions, Serology for hepatotropic viral infections hepatitis A,B,C,E., and sonography of the liver are desirable to exclude other causes of cholestasis. Laboratory test may show normal or mildly elevated conjugated serum bilirubin, normal to mildly increased transaminase, elevated serum alkaline phosphatase (partly of placental origin) negative serological markers and a normal liver on ultrasound examination.

\section{ASSOCIATED RISK}

Once thought to be benign for both mother and foetus, obstetric cholestasis has been associated with increased rates of fetal morbidity and mortality and increased risk of maternal cougulopathy.

\section{MATERNAL RISK}

Due to abnormalities in bile acid metabolism there is disruption of the absorption of fat soluble vitamins especially vit $\mathrm{k}$. This disruption leads to depletion of vit. k-dependent clotting factors and an increase in the rate of post-partum haemorrhage. $^{8}$

\section{FETAL RISK}

The foetus of an affected mother is at increased risk of number of events:

- a higher rate of intrauterine death with quoted rates of 100 per 1000 for perinatal mortality.

- Pernatal death is twice as common as still birth ${ }^{16}$ which usually occurs after 36 weeks.

- $\quad$ an increase in the no. of intrapartum events especially muconium stained liquor (25-50\%) and intra-partum fetal distress $12-22 \%$.

- a higher rate of spontaneous preterm delivery $12-44 \%$.

- $43 \%$ of the new born have birth weight less than $2.5 \mathrm{~kg}$

- a higher rate is intracranial haemorrhage secondary to vit $\mathrm{k}$ deficiency. ${ }^{16}$

- fetal coagulopathy resulting in intra arrythmias during fetal heart monitoring and muconium stained liquor, intracranial haemorrhage has been described. ${ }^{17}$

The mechanism where by the foetus is affected are unclear. There may be direct toxic effect from bile acids crossing the placenta and disrupting fetal physiology. ${ }^{5}$ Bile acids may have a direct vasoactive effect on the placental circulation. ${ }^{14}$ The risk to the foetus however donot correlate with maternal symptomatology or biochemistry. ${ }^{5}$ The exact risks to the foetus are difficult to determine as current management is to deliver the foetus at 37-38 weeks of gestation which has reduced perinatal mortality rates to $2-4 \% .{ }^{17}$ Fetal coagulopathy resulting in intracranial haemorrhage, arrythmias during fetal heart monitoring and muconium staining of amnico fluid in over $50 \%$ of the new born and altered composition of muconium in new born of mothers with ICP have been reported. ${ }^{17}$ The concentration 
of TBA and cholic acid in the muconium of babies born to mothers with ICP was much higher than in muconium of babies born of normal pregnancies. ${ }^{17}$

\section{MANAGEMENT}

The management goals for a pregnant women with obstetric cholestasis should include exclusion of other causes of cholestasis, relief of itching and jaundice, correction of coagulopathy and ensuring a good fetal outcome. The management can be divided into four categories: diagnosis, fetal surveillance, drug therapy and delivery planning.

\section{DIAGNOSIS}

Diagnosis is one of exclusion. Information on previous pregnancies, family history or associated problems while taking the oral contraceptive pill should be sought. Similarly pre-existing liver disease, intravenous drugs or alcohol abuse, other concommittant drug use e.g. methyl dopa and other risk factors for viral hepatisis should be identified. Investigation involve estimation of liver function. There is an alteration in normal liver function in the pregnant state (table I).

\section{Table I}

\section{Normal ranges for liver enzymes in non-pregnant and pregnant populations} (reproduced with permission ${ }^{16}$ )

\begin{tabular}{|l|c|c|c|c|}
\hline \multicolumn{1}{|c|}{ Liver enzyme } & Non- & \multicolumn{3}{c|}{ Trimester } \\
\cline { 3 - 5 } & pregnant & First & Second & Third \\
\hline Asparatate transaminase $(\mathrm{iu} / \mathrm{l})$ & $7-40$ & $10-27$ & $10-28$ & $11-29$ \\
\hline Alaninie transaminase $(\mathrm{iu} / \mathrm{l})$ & $0-40$ & $6-30$ & $6-29$ & $6-28$ \\
\hline Bilirubin $(\mu \mathrm{mol} / \mathrm{l})$ & $0-17$ & $4-15$ & $3-12$ & $3-14$ \\
\hline$\gamma$-Glutamyltransferase $(\mathrm{iu} / \mathrm{l})$ & $11-50$ & $5-37$ & $5-43$ & $3-14$ \\
\hline Alkaline phosphatase $(\mathrm{iu} / \mathrm{l})$ & $30-130$ & $32-100$ & $43-135$ & $133-418$ \\
\hline
\end{tabular}

A raised alkaline phosphatase is of little use due to the pregnancy associated rise. The typical pattern of abnormality in obstetric cholestasis is a two to four fold rise in Transaminase. There may also be a mild elevation of bilirubin level. In ICP. Serum bilirubin levels are known to be generally less than $6.0 \mathrm{mg} \%$. Total bile acid level shows a 10-100 fold increase. This investigation is particularly useful in the presence of normal level of Transaminase and other liver function tests when obstetric cholestasis is suspected. Other causes of cholestasis should be excluded as already mentioned. An ultrasound scan to exclude gall stones (although the presence of gall stone doesnot necessarily mean that these are the cause of the cholestasis, hepatitis serology including $\mathrm{ABC}$, cytomegalovirus and Epstein Barr virus, and a screen for auto immune liver disease such as chronic active hepatisis (anti smooth muscles antibodies) and primary biliary cirrhosis (antimitochondrial anti-bodies) should be performed.

\section{FETAL SURVEILLANCE}

Prediction of fetal compromise in this condition is difficult. Cardiotocography (CTG) monitoring of the foetus and umbilical artery doppler wave form studies are both used but are not usually found to be abnormal. ${ }^{18}$ Some authors recommended the use of anmiocentesis to detect muconium staining. ${ }^{16}$ The situation is analogous to the problems of predicting compromise in the foetus of the diabetes mother. Estimation of fetal growth (alternate weeks) and estimation of liquor volume and umbilicall artery doppler wave form studies normally performed weekly with least twice weekly CTG. Maternal and/or obstetrician anxiety may lead to admission for close monitoring.

\section{DRUG THRAPY}

Several medications have been tried for the relief of itching in the mother. Symptomatic relief may be achieved by the use of antihistamines, chlorpheniramine, terfenadine and promethazine have all been used for this purpose. However in most cases this basic treatment is insufficient. Pruritis in Cholestasis is of central origin and thus 
fails to respond to antihistamines and calamine, the commonest medicines to prescribe. A bile acid binding resin, cholestyramine has been shown to provide some relief in itching as has been the experience with charcoal and S-adenosyl methionine (SAM) These drugs have however not proved useful in correcting the others maternal abnormalities or improving fetal outcome. ${ }^{19}$ These drugs are also poorly tolerated and increases the relative risk of vit.k deficiency. ${ }^{22}$

Ursodeoxycholic acid (UDCA) an exogenous bile acid is increasingly being used $8-12 \mathrm{mg} / \mathrm{kg}$ in $\mathrm{BiD}$ doses. In clinical studies it has improved both pruritis and liver function. ${ }^{20}$ This drug is a friendly Hydroxyphilic bile acid which is also naturally present in the normal human bile albiet in small amount. UDCA tends to reset the abnormal bile acid ratio in ICP by replacing the toxic hydrophilic bile acids by the non-toxic hydrophilic ones. It also tend to increase bile flow by acting as a choleretic agent and stabilises the hepatocytes membrane protecting it from this noxiois effects of the toxic bile acids. There are several clinical studies which have shown that UDCA given to women with ICP in doses of $10-14 \mathrm{mg} / \mathrm{kg} /$ day in divided oral doses is not only safe but is extremely useful. There is marked reduction in itching and a decreased in Transaminase and bilirubin level. The clinical improvement was associated with a significant fall in the elevated TBA and CA levels. ${ }^{20,21,22}$ Even more interesting was the observation that similar favourable changes were observed in the foetus as well. There was a significant fall in the level of toxic bile acids in the muconium of these infants.

Dexamethasone has also been reported to be effective in reducing pruritis by decreasing the level of bile acids in the serum and thus improving the outcome as well. It has been shown to supress the fetal adrenal production of dehydro epiandrosterone sulphate (DHES) thus decreasing it conversion to oestrogen by the placenta. Supression of the high oestrogen level has been shown to decrease the level of bile acids and thus reduces pruritis. The doses of dexa methasone known to be effective in this condition is $12 \mathrm{mg}$ daily for 7 days tapered over a period of a few days. As Dexamethasone has been extensively used in premature babies to accelerate lung metauration, its safety for use in pregnancy is well established. ${ }^{23}$

\section{PLAN FOR DELIVERY}

Delivery is normally planned for 37-38 weeks of gestation. It may be appropriate earlier if maternal or fetal well being is in question. This may reduce perinatal mortality. ${ }^{5}$ Because of the increased rate adverse of intrapartum events, close monitoring is appropriate. Active management of third stage is necessary because of the increased risk of postpartum haemorrhage.

\section{POST NATAL}

Symptoms usually resolve soon after delivery although rarely they may worsen in the puerperium. Recurrence in subsequent pregnancies was initially thought to be in the order of $50 \%$ but figure of $60 \%-100 \%$ are more realistic. Use of the combined oral contraceptive pill should be discouraged but the progesterone only pill could be an alternative. As doses of oestrogen are physiological, normal hormone replacement therapy should not cause problems, except in some women with a history of cyclical itching.

\section{CONCLUSION}

Pruritis of pregnancy seems fairly common among pregnant women. It is also possible that ICP is responsible for a large number of unexplained still births especially after 36 weeks of gestation. A large prospective study is needed to address the problems of this condition, its epidemiology and its outcome. In the mean time, it is important to realise that by increasing awareness among the health personnel caring for the pregnant women that pruritis of pregnancy is a high risk condition and that close fetal monitoring and timed early intervention can 
decrease perinatal mortality significantly. Ursodeoxycholic acid seems to produce good clinical relief to the mother, corrects the disproportion of bile acids in the liver and blood and provides a favourable outcome to the foetus.

\section{REFERENCES}

1. Rayne - Fenning N, Kilby N. Obstetric cholestasis Fetal and maternal medicine 1997; 1-17.

2. Reyes H, Gonzales Mc, Ribatta J. Prevalence of intrahepatic cholestasis J. Prevalence of intrapatic cholestasis of pregnancy i chile AnnIntern ed 1978;88:487-93.

3. Burg B, helmg, Petersonl et al. Cholestasis of pregnancy. Clinical and laboratory studies. Acta obstet gynaecol scand 1986; 65:107-13.

4. Waine, $C$. Beware of itching during late pregnancy. Practioner 1995:239:97-100.

5. Reyes H. The spectrum of liver and gastrointestinal disease in cholestasis of pregnancy. Gastroenterol lin North Am 1992;905-21.

6. Hirvioja MI, Kinnes. Inheritance of intrahepatic cholestasis of pregnancy in one kindred clin genot 1993;43:315-17.

7. Williamson $\mathrm{C}$, Froome $\mathrm{Va}$, forbes et al. A study of the clinical features and establishment of the genetic aetiology of obstetric cholestasis in a united kingdom cohort. J . obst gynaecol 1998; 18 supp II; s14-s15

8. Fagon EA. Disorders of liver, biliary system and pancreas. Medical disorder in obstetrc practice, de swiet M. oxford, Blackwell science. 1995,331-33.

9. Dabidson KM, Intraheptic cholestasis of pregnancy. Seminars in perinatology 1998; 22(2): 104-11.

10. Gonzalez M, Reyes M, Figure OD et al. Intrahepatic cholestasis of pregnancy in twin pregnancy. Hepatology 1989; 9: 84-90.

11. Erfling W. Effect of oestrogens on the Liver. Gastroenterology 1978;75:512-22.

12. Schrciber AJ, Simon FR.Oestrogen induced cholestasis; clues to pathogenesis and treatment. Hepatology 1983; 3:607-13.
13. Brites $\mathrm{D}$, Rodrigues CM, Van-Zeller $\mathrm{n}$, Brito A Silva R. Relevance of serum bile acid profile in the diagnosis of intrahepatic cholestasis of pregnancy in a high incidence area: Portugal and European J ournal of obstetrics, Gynaecology and reproductive Biology. 1998; 80 (1) : 31-8.

14. Elias E. Jaundice. Oxford Text Book of medicine Vol I Weatherall, Ledingham warell ELBS 1988, 12; 221-212.

15. Jones $E$, Bergasa N. The pruritis of cholestasis from bile acids to opiate antogonists. Hepatology 1990; 11:884-7.

16. Fisk NM, storey GNB. Fetal outcome in obstetric cholestasis Br. J. Obstet Gynaecol 1988; 95:1137-1143.

17. Reo Seco Aj, Ivanovic MB, Manzura et al. Intrahepatic cholestssis of pregnancy: a refruspective case control study of perinatal outcome. AM. Jr of obstet and gynaecol 1994;170(3) :890-5.

18. 2 immerman P, Koskinen J, Vaglamo P, Ranta J . Doppler umbilical artery velocimetry in pregnancies complicated by ICP. J. Perinatal Med. 1991;19:351-5.

19. Florieni A, Paternoster D, Melis A, Grella PV et al .S-Adenosmetionnine versus ursodeoxycholic acid in the treatment of intrahepatic cholestasis of pregnancy. Europen J r. of Obstetrics gynaecology and Reproductive biology. 1996;67(2):109-13.

20. Palma J, Reyesh,Ribatta J et al. Effects of ursodeoxy cholic acid in patients with intrahepatic cholestasis of pregnancy hepatology 1992; 15:1043-7.

21. Serrano MA, Brites D, Larena MG et al. Beneficial effect of ursodeoxy cholic acid on alterations induced by cholestsis of pregnancy in bile acid transport across the human placenta. Jr. of hepatology 1998 2815] :829-39.

22. David $M H$, de silva RC, Jones SR et al. Fetal mortality associated with cholestasi of pregnancy and the potential benifit of thepary with ursodeoxy cholic acid. Gut 1995;37(4) 580-4.

23. Hirvioja MI, Tuimala R, Vuori J, The treatment of intrahepatic cholestasis of pregnancy by dexamethasone. Br. J. Obsr. And Gynae 1992; 99: 109-111. 